\title{
Enlargement of the bony orbit by orbital recurrence of choroidal melanoma 21 years after enucleation
}

\author{
HUIBERT JAN SIMONSZ ${ }^{1 *}$ and KARIN LÖFFLER ${ }^{2}$ \\ 'Department of Strabismus \& Neuro-ophthalmology, Eye Clinic, Kantonsspital, Sankt-Gallen, \\ Switzerland, and the ${ }^{2}$ Department of Ophthalmo-pathology, University Eye Clinic, Freiburg, \\ Germany
}

\begin{abstract}
A case is presented of a 58-year-old retarded male with a $6 \mathrm{~cm}$, painless, hard, pigmented tumor filling the left orbit completely, after enucleation 21 years previously for retinal detachment, glaucoma and no light perception. CT scan and MRI revealed, besides the tumor, an outspoken enlargement of the bony orbit with thinning of the orbital walls. A biopsy showed a spindle B cell melanoma. In one of the paraffin histology sections of the globe enucleated 21 years previously a very small spindle B cell melanoma under the detached retina was present, with tumor cells in several vortex veins. The tumor was debulked centrally and it was exenterated and the eyelid skin was closed over the empty orbit. The patient is well 15 months after surgery. This case re-emphasizes that a choroidal melanoma can recur locally decennia after enucleation, that the histology of the tumor may change concomitantly and that a slowly growing orbital tumor can enlarge the bony orbit without perforating the periborbit or eroding the bone.
\end{abstract}

Key words: orbital tumor; choroidal melanoma ; orbital anatomy ; exenteration

Local recurrences of choroidal melanoma have been described to occur 24, 28 and even 42 years after enucleation ${ }^{1-3}$. When allowed to grow over an extended period, such tumors may enlarge the bony orbit considerably.

A 58-year-old retarded male presented with a painless, hard, pigmented tumor filling the left orbit completely (Fig. 1). An enucleation had been performed 21 years previously because of retinal detachment, glaucoma and no light perception. One year previously, the nurse caring for the patient had noticed that the patient's prosthesis tended to fall out, and she had a smaller prosthesis made. Nine months later the new

* Correspondence to: H.J. Simonsz, M.D., Department of Strabismus \& Neuro-ophthalmology, Eye Clinic, Kantonsspital, Sankt-Gallen, CH-9007 Switzerland. prosthesis also started to fall out and ophthalmological advice was sought. CT scan and MRI

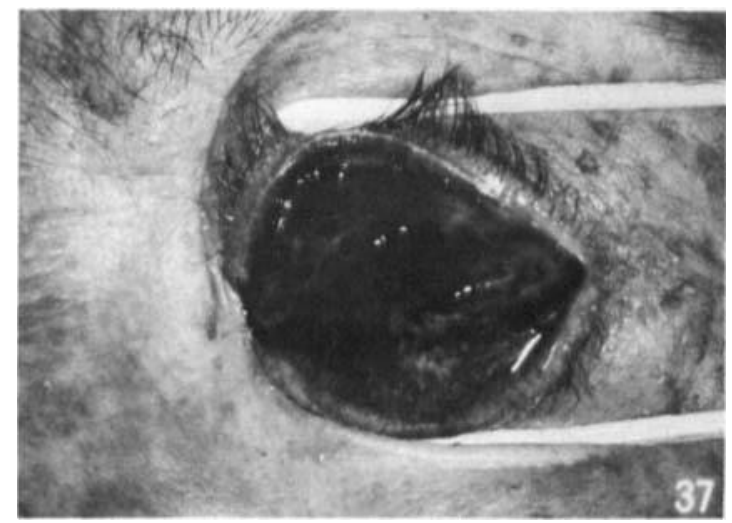

Fig. 1. A painless, hard, pigmented tumor filled the left orbit completely; an enucleation had been performed 21 years previously. 


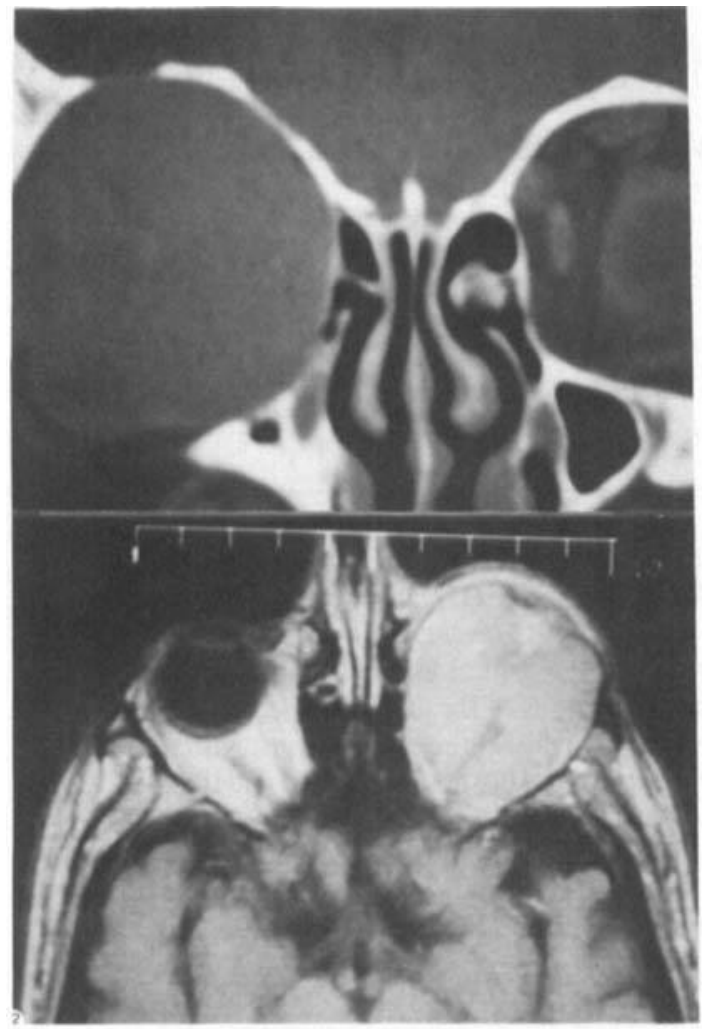

Fig. 2. CT scan (a) and MRI (b) shows a large tumor with areas of necrosis and with enlargement of the bony orbit.

(Fig. 2) revealed a tumor, $6 \mathrm{~cm}$ in diameter, with areas of necrosis and with enlargement of the bony orbit. We took a transconjunctival biopsy from the tumor. Macroscopically, it had brightly colored orange-yellow areas, in addition to dark, pigmented areas and areas with necrosis. Histologic examination (Fig. 3) showed a malignant melanoma consisting mainly of spindle B cells with some epitheloid cells, with areas of necrosis and hemorrhage, and with occasional giant cells. In the necrotic areas, iron deposits and pigment with autofluorescence were found. Mitoses were rare and a lymphocytic infiltration was lacking. The paraffin histology sections of the globe enucleated 21 years previously were obtained and in one of the 25 sections a very small malignant melanoma under the detached retina was present, consisting mainly of spindle A cells, with some spindle B cells (Fig. 3). In the same section, there were tumor cells in several vortex veins, signifying transscleral extension which, together with the large retinal detachment, would be compatible with a diagnosis of diffuse melanoma. Chest X-ray films, sonography of the abdomen, liver scintigraphy and bone scintigraphy showed no metastasis.

We exenterated the orbital contents: First the tumor was debulked centrally and continuous suction was applied by a temporary drain. Debulking the tumor increased room for preparation of the periorbit off the bone, which had to be done carefully, without perforating the very thin bone. Suction was intended to avoid renewed distention of the tumor by filling with blood, and to reduce, possibly, blood-borne metastases. A different set of instruments was used for the rest of the procedure to avoid tumor cell spilling outside the periorbit. The exenteration was not difficult: The periorbit was not adherent to the bone, and the bony membrane was intact everywhere. After exenteration, the eyelid skin, which had been undermined and separated from the eyelids beforehand, was closed over the empty orbit. The postoperative course was uneventful. After the skin sunk into the orbit, over a deep hematoma and the wound was healed, radiation was initiated. A control CT scan showed a hematoma without contrast enhancement. The patient is well 15 months after surgery.

This case emphasizes (a) that a choroidal melanoma can recur locally decennia after enucleation, (b) that the histology of the tumor may change concomitantly and (c) that a slowly growing orbital tumor can enlarge the bony orbit without perforating the periorbit or eroding the bone. 


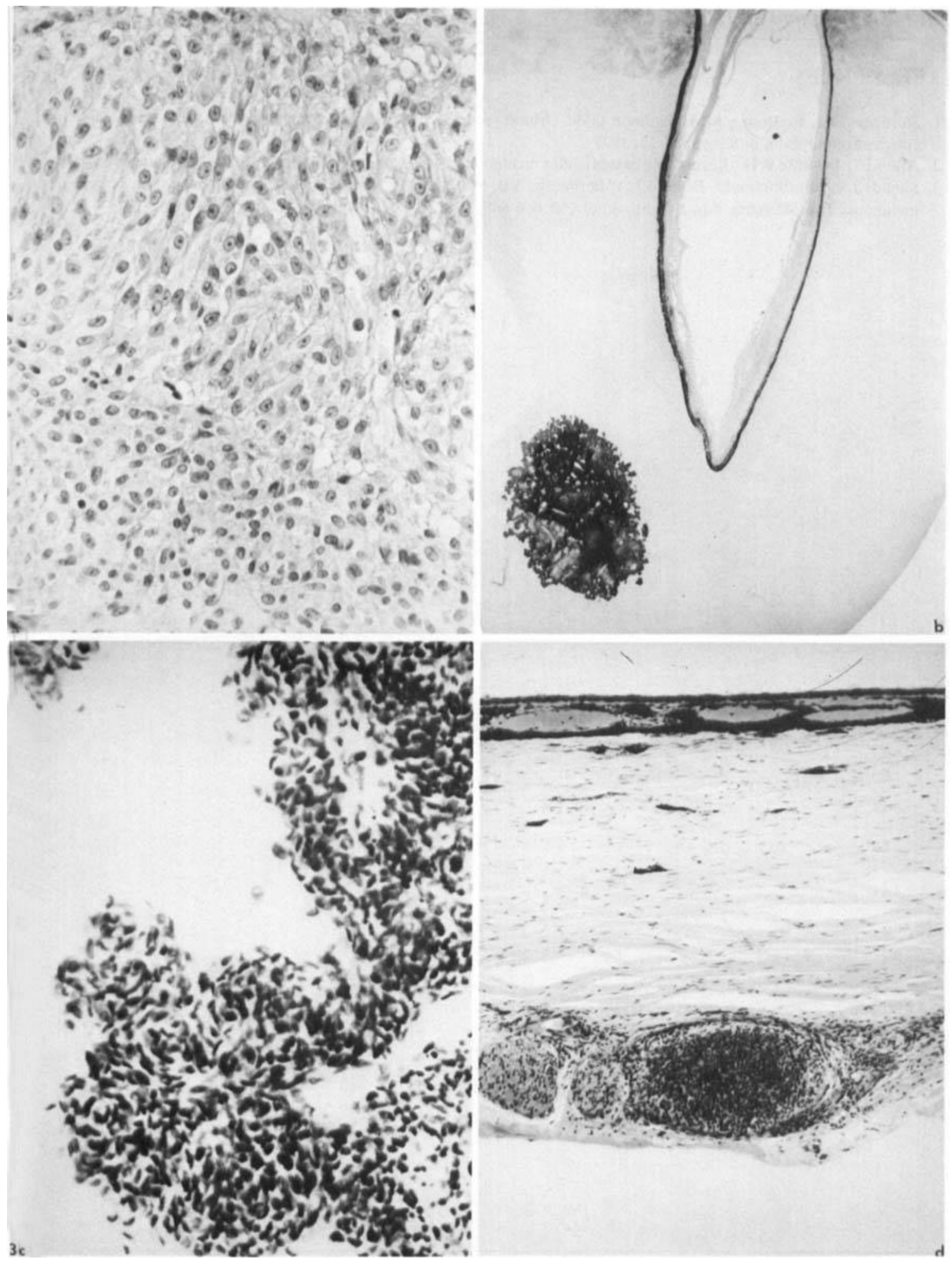

Fig. 3. Histology (a) shows a malignant melanoma consisting mainly of spindle B cells with some epitheloid cells; in one of the 25 sections of the globe enucleated 21 years previously (b) a very small malignant melanoma under the detached retina was present (arrow), consisting (c) mainly of spindle A cells, with some spindle B cells, while (d) infiltrating a vortex vein. 


\section{REFERENCES}

1. Saunders DH, Rodrigues MM, Shannon GM: Orbital recurrence of malignant melanoma of the choroid 24 years after enucleation. Ophthalm Surg 8:31-35, 1977

2. Allen JC, Jaeschle WH: Recurrence of malignant melanoma in the orbit after 28 years. Arch Ophthalmol 76:79-81, 1966

3. Shields JA, Augsburger JJ, Donoso LA, Bernadino VB, Portenar M: Hepatic metastasis and orbital recurrence of uveal melanoma after 42 years. Am J Ophthalmol 100:666-668, 1985 